\title{
CARTAS AL EDITOR
}

\section{Factores de riesgo cardiovascular modificables en los médicos y en otro personal del equipo de salud}

Señor editor: La enfermedad cardiovascular (ECV) constituye un grave problema de salud pública mundial por ser la primera causa de morbilidad y mortalidad en varios países. ${ }^{1}$ El desarrollo de dicha enfermedad es promovido por varios factores de riesgo, algunos de los cuales son modificables y sujetos a medidas preventivas. ${ }^{2}$ Aunque los factores de riesgo modificables de ECV tienen adecuadas estrategias de prevención, los médicos y el equipo de salud, en general, no intervienen adecuadamente en este aspecto con sus pacientes. Pocos enfermos son advertidos de los beneficios del control de peso, la presión arterial, la concentración de la glucosa y los lípidos; existe, por lo tanto, una aparente desconexión entre la evidencia clínica y la práctica médica, que parece incrementarse cuando los médicos o el personal del equipo de salud son los pacientes. ${ }^{3}$ Con base en lo anterior, tratamos de identificar la frecuencia de factores de riesgo cardiovascular modificables en los médicos y en otro personal del equipo de salud. Para ello se llevó a cabo un estudio transversal analítico en 264 voluntarios médicos y otro personal del equipo de salud (dentistas, enfermeras, promotores, psicólogos, químicos, terapistas físicos y trabajadoras sociales) de 23 estados de la República Mexicana durante el Congreso Nacional de Salud Pública en el año 2004, con una edad entre
20 y 65 años. El IMC fue clasificado de acuerdo con los siguientes valores: bajo peso con menos de $18.5 \mathrm{~kg} / \mathrm{m}^{2}$; normal 18.5 a $24.9 \mathrm{~kg} / \mathrm{m}^{2}$; sobrepeso 25.0 a 29.9 $\mathrm{kg} / \mathrm{m}^{2}$; obesidad $\geq 30.0 \mathrm{~kg} / \mathrm{m}^{2}$. Se consideró como un valor de riesgo una cintura mayor o igual a $90 \mathrm{~cm}$ (hombres) y mayor o igual a $80 \mathrm{~cm}$ (mujeres). La tensión arterial fue clasificada como normal abajo de $120 / 80 \mathrm{~mm} \mathrm{Hg}$, como prehipertensión arterial 120-139/80-89 mm Hg e hipertensión arterial con mayor o igual a 140/90 mm Hg. Los exámenes de laboratorio se realizaron después de un ayuno de 10 a 12 horas. La glucemia capilar se determinó por medio de un análisis electroquímico glucosa-oxidasa (OneTounch Ultra, LifeScan, Johnson \& Johnson Co, Milpitas, $\mathrm{Ca}$, EUA). Se consideró como una glucemia normal un valor menor a $5.6 \mathrm{mmol} / \mathrm{l}$, glucosa de ayuno anormal de 5.6 a $6.9 \mathrm{mmol} / \mathrm{l}$ y como diabetes mellitus uno mayor o igual a 7.0 $\mathrm{mmol} / \mathrm{l}$. El colesterol se midió con una técnica de fotometría reflectante (Accutrend GC, Diavant, Roche Diagnostics, Basilea, Suiza) y fue clasificado como normal un valor menor de $5.2 \mathrm{mmol} / \mathrm{l}$ y como factor de riesgo uno mayor o igual a $5.2 \mathrm{mmol} / \mathrm{l}$. Los sujetos también fueron agrupados de acuerdo al número de factores de riesgo presentes.

Del total de individuos estudiados, $140(53.0 \%)$ pertenecieron al género masculino y $124(47.0 \%)$ al femenino, de los cuales $178(67.4 \%)$ fueron médicos y 86 $(32.6 \%)$ correspondieron a otro personal del equipo de salud. Los médicos presentaron estadísticamente, en comparación con el resto del personal del equipo de salud, una mayor edad ( $44.8 \pm 8.7 \mathrm{vs} .42 .2$ \pm 8.0 años, $p=0.021)$, IMC ( $28.6 \pm 4.1$ vs. $\left.27.5 \pm 4.4 \mathrm{~kg} / \mathrm{m}^{2}, p=0.044\right)$ y tensiones arteriales sistólica (121 \pm 14 vs. $116 \pm 12$ $\mathrm{mm} \mathrm{Hg}, p=0.001)$ y diastólica $(82 \pm 8 v \mathrm{vs}$. $78 \pm 8 \mathrm{~mm} \mathrm{Hg}, p=0.006)$. La hipertensión arterial fue más frecuente en los médicos que en el resto del equipo de salud (41.0 vs. $17.4 \%, p<0.001)$. En los médicos fue más frecuente la presencia de 3 y 4 (26.8 vs. $9.8, p=0.001$ y 21.5 vs. 9.4, $p=0.017$, respectivamente) factores de riesgo cardiovascular modificables que en el resto del personal del equipo de salud.

Los médicos y el equipo de salud en general tienen un amplio conocimiento sobre la modificación de los factores de riesgo; sin embargo, existe evidencia de que durante la práctica clínica rutinaria, frecuentemente de forma sistemática se pierde la oportunidad de su prevención en la población, por lo que existe una amplia brecha entre el conocimiento de los factores de riesgo por parte del médico y la efectividad de los resultados del consejo preventivo en su práctica clínica. ${ }^{4}$ Las diferentes actitudes en los médicos frente a los factores de riesgo cardiovascular pueden hacer variar el impacto de intervención en los pacientes. ${ }^{5} \mathrm{La}$ alta frecuencia de factores de riesgo encontrados en nuestro estudio demuestra la falta de aplicación del conocimiento médico en la salud del propio médico. Es imperativo encontrar estrategias para modificar las actitudes y la aplicación del conocimiento médico en la salud del propio médico con el 
propósito de prevenir la ECV que presenta un incremento de proporciones pandémicas.

Dr. Agustín Lara Esqueda, (I) Dr. Oscar Velázquez Monroy, ${ }^{(I), t}$

Dr. Cuauhtémoc Ruiz Matus, ${ }^{(2)}$ Dra. en C. Esperanza Martínez-Abundis, ${ }^{(3)}$ Dra. Isidora Guadalupe Valera-González, ${ }^{(3)}$ Dr. en C. Manuel González-Ortiz. ${ }^{(3,4)}$

'Centro Nacional de Vigilancia Epidemiológica y Control de Enfermedades, Secretaría de Salud. ${ }^{2}$ Sociedad Mexicana de Salud Pública. ${ }^{3}$ Unidad de Investigación Médica en Epidemiología Clínica, Hospital de Especialidades, Unidad Médica de Alta Especialidad, Centro Médico Nacional de Occidente, Instituto Mexicano del Seguro Social. ${ }^{4}$ Departamento de Fisiología, División de Disciplinas Básicas, Centro Universitario de Ciencias de la Salud, Universidad de Guadalajara. t Finado.

Montes Urales 1409, Col. Independencia, 44340 Guadalajara,Jalisco. Correo electrónico: uiec@prodigy.net.mx

\section{Referencias}

I. Levenson JW, Skerrett PJ, Gaziano JM. Reducing the global burden of cardiovascular disease: the role of risk factors. Prev Cardiol 2002;5:188-199.

2. Eaton CB. Traditional and emerging risk factors for cardiovascular disease. Prim Care 2005;32:963-76.

3.Tsui JI, Dodson K, Jacobson TA. Cardiovascular disease prevention counseling in residency: resident and attending physician attitudes and practices. J Natl Med Assoc 2004;96:1080-1083; 1088-1091.

4. Castaldo J, Nester J, Wasser T, Masiado T, Rossi M,Young M, et al. Physician attitudes regarding cardiovascular risk reduction: the gaps between clinical importance, knowledge, and effectiveness. Dis Manag 2005;8:93-105. 5. Grant AM, Niyonsenga T, Dion I, Delisle E, Xhignesse M, Bernier R. Cardiovascular disease. Physician attitudes toward prevention and treatment. Can Fam Physician 1998;44:780-787.

\section{Contaminación y salud pública en México}

Señor editor: al abordar el tema de la contaminación ambiental debemos considerar no sólo las fuentes antropogénicas sino también a las fuentes naturales de contaminación, tal es el caso de la producción de metano en zonas pantanosas, las tormentas eléctricas que generan ozono y óxidos de nitrógeno, la actividad animal que genera bióxido de carbono e hidrogeniones, la erosión, deforestación y sequías que generan partículas esparcidas por el viento y la actividad volcánica que emite óxidos de azufre y partículas. $\mathrm{Al}$ respecto podemos percatarnos que son pocos los trabajos que reportan investigación respecto a los efectos de la contaminación por fuentes naturales en salud pública. De tal forma el grueso de trabajos se refieren a los efectos por la contaminación antropogénica a diferentes niveles de organización (molecular, celular, órganos, organismos, poblaciones, comunidades y/o ecosistemas).

Si hablamos a nivel nacional nos percatamos que esta búsqueda de trabajos se reduce aun más respecto a dicha problemática, haciendo énfasis que México presenta problemas de contaminación considerables, principalmente en las ciudades con un creciente desarrollo urbano, industrial, tecnológico y actividad volcánica durante los últimos doce años, en el caso del Popocatépetl. Ante dicho escenario para lograr una buena calidad del medio ambiente se depende de factores económicos, políticos y socioculturales, además de la coordinación de diferentes sectores como salud, educación, ecología, desarrollo urbano, obras publicas, energía, industria, transporte, iniciativa privada y población en general.

Mientras los países desarrollados se preparan para enfrentar el problema de la contaminación, tratando de minimizar los impactos en la salud, mediante la mejoría y expansión de los servicios de salud, preparándose para enfrentar desastres y mejorando la tecnología disponible para crear ambientes propicios para la población, ${ }^{2}$ sólo queda preguntarnos: ¿en México qué estamos haciendo al respecto?, por tal motivo es importante destacar el trabajo de los diversos grupos de investigación que en nuestro país se están dando a la tarea de estudiar los efectos que involucran la contaminación respecto a salud pública y que han sido publicados en la revista Salud Pública de México, donde consideran los siguientes temas: las condiciones geológicas de la zona metropolitana de la Ciudad de México, las condiciones que favorecen la persistencia de contaminantes en la atmósfera y sus efectos sobre la población. De la relación entre las concentraciones ambientales de partículas $\mathrm{PM}_{10}$ y de ozono con el número diario de consultas al servicio de urgencias por enfermedades respiratorias agudas y asma en población menor de 15 años, y de la prevalencia y severidad del asma, de la rinitis y del eczema en escolares. ${ }^{3-6}$

Debido a que la actividad volcánica está de "moda" en la región central del país y aporta una cantidad considerable de contaminantes, principalmente a la atmósfera, se ha documentado que dicha actividad representa riesgos para los ecosistemas y las poblaciones humanas que se ubican cerca de los edificios volcánicos; no obstante se ha descrito que incluso organismos que se localizan a distancias considerables de las zonas con actividad volcánica también pueden verse afectados. ${ }^{7-10}$ Puesto que la ceniza volcánica está constituida principalmente por dióxido de azufre, este compuesto puede producir irritación local y desarrollar silicosis y en los pacientes con hiperreactividad bronquial, asma o enfermedades pulmonares obstructivas crónicas, la exposición a las cenizas puede complicar la enfermedad. A nivel de la conjuntiva, la ceniza actúa como un cuerpo extraño, siendo los cristales de dióxido de azufre los que afectan directamente a la conjuntiva y a la córneas, produciendo abrasiones, además del efecto irritante. Por su parte, los microelementos presentes en la ceniza, como el bromo volcánico, puede formar parte del agua de vertientes y durante la potabilización generar trihalometanos, que son compuestos cancerígenos. De tal forma, los principales riesgos volcánicos involucran la emisión de ceniza y gases, relacionándose con la cantidad y el número de exposiciones a dichos eventos. En este contexto, la colaboración entre 